\section{Ranking of cadmium low amount measurement systems according to economic, environmental, and functional indicators using ELECTRE analytical method}

\author{
Benyamin Chahkandi', Mohammad Gheibi ${ }^{2 *}$ and Amir Takhtravan ${ }^{3}$ \\ ${ }^{1}$ Department of Civil Engineering, University of Tehran, Iran \\ 2Department of Civil Engineering, Ferdowsi University of Mashhad, Mashhad, Iran \\ ${ }^{3}$ Department of Civil Engineering, Birjand University of Technology, Iran
}

\section{Abstract}

Cadmium is one of the transition metals, known by the scientific name $\mathrm{Cd}$. One of its main characteristics is the high toxicity, even in very little amounts. Cadmium is often released through industrial effluents, pesticides, chemical fertilizers, and the burning of fossil fuels. Since the presence of cadmium ions in the living organisms' body, especially humans, can cause serious damage to the liver and pancreas, and also because its role in causing cancer has been proven, measuring very low amounts of this metal is of high importance. In the first step, this study has reviewed and analyzed common laboratory methods for measuring small amounts of cadmium. Then, according to economic, environmental, feasibility, speed, and accuracy factors, all available methods were evaluated using the ELECTRE technique. The results showed that the extraction methods using Dowex Optipore V-493 resin and extraction system in Triton X-114 surfactant, placed in the first and second positions.

\author{
More Information \\ *Address for Correspondence: \\ Mohammad Gheibi, Department of Civil \\ Engineering, Ferdowsi University of Mashhad, \\ Mashhad, Iran, \\ Email:mohamadgheibi@ymail.com \\ Submitted: August 10, 2021 \\ Approved: September 07, 2021
}

Published: September 08, 2021

How to cite this article: Chahkandi B, Gheibi M, Takhtravan A. Ranking of cadmium low amount measurement systems according to economic, environmental, and functional indicators using ELECTRE analytical method. Ann Civil Environ Eng. 2021; 5: 003-006.

DOI: 10.29328/journal.acee.1001028

Copyright: @ 2021 Chahkandi B, et al. This is an open access article distributed under the Creative Commons Attribution License, which permits unrestricted use, distribution, and reproduction in any medium, provided the original work is properly cited.

Keywords: Cadmium; Decomposition methods; Economics; Environment; ELECTRE

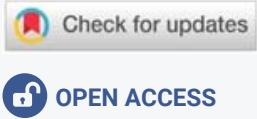

\section{Introduction}

Today, environmental pollution is one of the most complex challenges of communities, environmental management, and control systems. Among these pollutants in water, soil, and air, the existence of different concentrations of heavy metals has to be mentioned. Meanwhile, among all heavy metals, cadmium is of special importance due to its acute and chronic epidemiological effects [1]. Low concentrations of cadmium are found at about $0.2 \mathrm{mg} / \mathrm{kg}$ of the lithosphere, $0.53 \mathrm{mg} / \mathrm{kg}$ of the soil surface, and $0.66 \mathrm{mg} / \mathrm{kg}$ of the dry weight of the edible plants [2]. Cadmium can leak into the environment through solid waste and wastewater of industries such as; Electroplating, plastics production, mining, alloy production, pigments, and batteries [3]. The International Agency for Research on Cancer (IARC) has identified cadmium as a major carcinogenic agent as well as renal failure [4]. On the other hand, due to the biomagnification and the cumulative effect of small amounts of cadmium in the upper species of the food chain, the importance of measuring small amounts of cadmium in real samples becomes more and more obvious [5]. Separation methods include precipitation, crystallization, freezing, evaporation, distillation, liquid-liquid extraction, solid-phase extraction, droplet extraction, and so on. Due to the measurement limitations for low amounts of cadmium and the lack of decomposition devices, pre-concentration methods are popular. Due to the small amounts of analyte in the samples, different extraction methods have been employed for pre-concentration, each of which has advantages and disadvantages. In some methods, like liquid-liquid extraction long extraction time, and in some others, such as re-extraction along with solvent and activated carbon, a large volume of organic solvent and sample could be the disadvantage [6] Hence, in recent years, microextraction methods by low sample consumption have been welcomed [7]. The present study also intends to prioritize small cadmium detection systems using the ELECTRE ${ }^{1}$ hierarchical analysis method and considering the economic, environmental, feasibility, speed, and accuracy of measurement systems.

${ }^{1}$ ELimination Et Choice Translating REality 


\section{Materials and methods}

\section{Cadmium measurement methods}

Researchers and industries have used various methods to measure small amounts of cadmium. In this study, measurement methods and systems will be compared and evaluated based on economic, environmental, feasibility, speed, and accuracy indicators. These methods and systems are described in Table 1.

\section{Data analysis using ELECTRE method}

ELECTRE (Elemination and Choice Expressing Reality) method is a multi-decision method first proposed by Benayoun and Roy in 1966. It is based on binary superiority comparisons between alternative decision points for each rating factor [22]. In this method concordance and discordance indexes are defined as measurements of satisfaction and dissatisfaction for decision maker in choosing one alternative over another. These indexes are then used to analyze the outranking relations among the alternatives [23] using following 8 steps [24].

1. Construct a decision matrix

2. Construct the normalized decision matrix

3. Calculate the weighted normalized decision matrix

\section{Ascertainment of Concordance to Discordance set}

5. Calculation of The Concordance and Discordance Matrices
6. Determine The Concordance and Discordance Dominance Matrix

7. Determine the aggregate dominance matrix

8. Eliminate the less favorable alternative and rank them

In this research, instead of ranking the options, we use a new concept called "non-ranking" $[25,26]$. For example, $A_{k} \rightarrow A_{l}$ means that the DM and the risk analyst accept that option $A_{k}$ is better than option $A_{t^{*}}$. First, with the help of Equation (1), we convert the decision matrix into a scaleless one.

$$
n_{i j}=\frac{r_{i j}}{\sqrt{\sum_{i=1}^{n} r_{i j}^{2}}}
$$

The matrix of the $S_{k l}$ concordance set and the $D_{k l}$ discordance set will be calculated following equation (2) then the criterion of concordance between $A_{k}$ and $A_{l}$ will be estimated based on equation (3) which the higher value of $I$ indicates the more appropriateness of $A_{k}$ rather than $A_{l}$.

$$
D_{k l}=\left\{j \mid r_{k j}<r_{l j}\right\} \quad S_{k l}=\left\{j \mid r_{k j} \geq r_{l j}\right\}
$$

$$
I_{k l}=\sum_{J \in S_{K L}} w_{j} ; \sum_{j=1}^{n} w_{j}=1
$$

The discordance matrix is formed using Equation (4) and the effective concordance matrix based on the minimum

\begin{tabular}{|c|c|c|}
\hline Methode & Researchers & description \\
\hline A1 & Jahromi et al. [8] & $\begin{array}{l}\text { In this method, after immediate injection of carbon tetrachloride extracting solvent, methanol dispersing solvent, and ammonium } \\
\text { pyrrolidine dithiocarbamate (APDC) ligand into a certain volume of an aqueous solution containing cadmium ion, the cadmium-APDC } \\
\text { non-polar complex is extracted in tetrachloride extracting solvent. }\end{array}$ \\
\hline A2 & Dadfarnia et al. [9] & $\begin{array}{l}\text { In this method, cadmium is reacted with anion I, which produces } \mathrm{CdI}^{2-} \text {, and to be able to extract the ionic complex into the organic } \\
\text { phase, the opposite ion of methyltrioctylammonium chloride was used. }\end{array}$ \\
\hline A3 & Souza Dias et al. [10] & In this method, bio sorbents modified with thiazol-resorcinol ligand (TAR) were used to pre-concentrate cadmium ions. \\
\hline A4 & Abbasi et al. [11] & $\begin{array}{l}\text { In this method, first cadmium and copper ions were reacted with a 3-aminophthalic hydrazide ligand and then the complexes formed on } \\
\text { the surface of the mercury droplet electrode were analyzed. }\end{array}$ \\
\hline A5 & Xiang et al. [12] & $\begin{array}{l}\text { In this method, cadmium with iodide anion produces Cdl42, which reacts with methyl green reagent to form a pair of non-polar ions that } \\
\text { can be extracted in Triton X-114 surfactant. }\end{array}$ \\
\hline A6 & Li et al. [13] & $\begin{array}{l}\text { In this method, after the formation of a complex between cadmium and diethyldithiocarbamate ligand, 1-hexyl-3-methyl imidazolium } \\
\text { hexafluorophosphate solvent was used as the extracting solvent. }\end{array}$ \\
\hline A7 & Melek et al. [14] & $\begin{array}{l}\text { In this method, after the reaction between cadmium and lead ions and dibenzyl dithiocarbamate ligand, Dowex Optipore V-493 resin } \\
\text { was used to extract the desired complex. }\end{array}$ \\
\hline A8 & Parham et al. [15] & The researchers used sulfur to pre-concentrate small amounts of lead and cadmium ions. \\
\hline A9 & Fang et al. [16] & In this method, mold adsorbent of thiol-activated silica ion was used for selective preconcentration of cadmium ions. \\
\hline A10 & Tuzen et al. [17] & $\begin{array}{l}\text { In this method, after mixing the mentioned metals with pyridylazo naphthol ligand, chromosorbent resin adsorbent was used to pre- } \\
\text { concentrate the complexes. }\end{array}$ \\
\hline A11 & Maranhao [18] & $\begin{array}{l}\text { In this method, after mixing the mentioned ions with a diethyl dithiophosphate ligand, the cloud point extraction method was used to pre- } \\
\text { concentrate the samples. }\end{array}$ \\
\hline A12 & Xiang et al. [12] & In this method, after creating an anion complex of cadmium ion and iodide ion, malachite green was used as the opposite ion. \\
\hline A13 & Mortada et al. [19] & $\begin{array}{l}\text { In this method, after creating a non-polar complex of cadmium ion and ligand 9.-10-phenanthroquinone-monoethylthio-toxic carbazide, } \\
\text { cloud point extraction based on micelle formation from Triton X-114 surfactant was used. }\end{array}$ \\
\hline A14 & Jafarvand et al. [20] & $\begin{array}{l}\text { In this method, after forming cadmium-ammonium pyrrolidine dithiocarbamate complex, a mixture of decanoic acid (extracting solvent) } \\
\text { dissolved in tetrahydrofuran dispersant solvent was quickly injected into an aqueous solution, resulting in a non-polar complex in the } \\
\text { decanoic acid extracting solvent. }\end{array}$ \\
\hline A15 & Zhang et al. [21] & $\begin{array}{l}\text { In this method, diethyl dithiophosphate was used as a complexing agent and after the formation of the complex, a mixture of carbon } \\
\text { tetrachloride in methanol was used to pre-concentrate the complex of the mentioned metals. }\end{array}$ \\
\hline
\end{tabular}
threshold of two binary array Boolean matrices named $\mathrm{F}$ and

Table 1: Methods and systems for measuring low amounts of cadmium in the present study. 
G, based on Equation (5).

$$
\begin{array}{ll}
N I_{k l}=\frac{\max _{j \in D_{K L}}\left|V_{k j}-V_{l j}\right|}{\max _{j \in J}\left|V_{k j}-V_{l j}\right|} \\
\bar{I}=\sum_{k=1 l=1}^{m} \sum_{k, l}^{m} / m(m-1) & \overline{N I}=\sum_{k=1 l=1}^{m} \sum_{k=1}^{m} N I_{k, l} / m(m-1) \\
\text { if }: I_{k, l} \geq \bar{I} \rightarrow f_{k, l}=1 & \text { if }: N I_{k, l} \geq \overline{N I} \rightarrow g_{k, l}=1 \\
\text { if }: I_{k, l}<\bar{I} \rightarrow f_{k, l}=0 & \text { if }: N I_{k, l}<\overline{N I} \rightarrow g_{k, l}=0
\end{array}
$$

Finally, the overall $h$ matrix, representing the order of relative preferences of the options, will be calculated using Equation (6).

$$
h_{k, l}=f_{k, l} \cdot g_{k, l}
$$

Finally, it should be noted that all of the above mathematical relationships have been implemented in the MATLAB 2012b programming environment.

\section{Results and discussion}

The analysis begins with scoring each parameter and studied options based on the five indicators of economic, environmental, feasibility, measurement speed, and accuracy. These scores have been obtained by asking from 9 experts in this field. The results are shown in Figure 1.

The ELECTRE calculations were performed on the above values. The results of the prioritization of the mentioned methods are summarized in Table 2 .

As shown in the table 2 Options A7 (Dowex Optipore V-493) and A5 (Triton X-114 surfactant) are the first and second positions in the low cadmium metering methods ranking. In this prioritization, economic, environmental, feasibility, measurement speed, and accuracy indices have weights of $0.25,0.2,0.1,0.15$, and 0.3 , respectively. On the other hand, options A7 and A5 have differences from other options in terms of these indicators. The distribution of the score difference between the A7 option and the other options

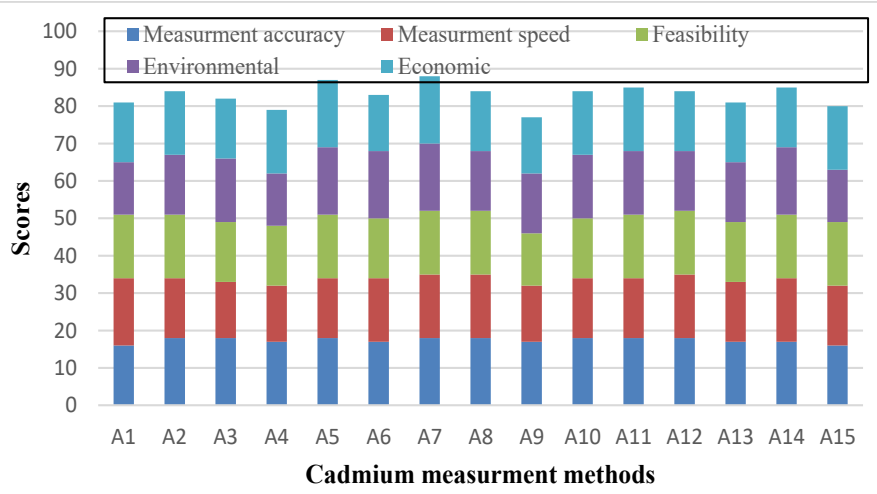

Figure 1: Scoring values for different cadmium measurement options based on five normalized indices.

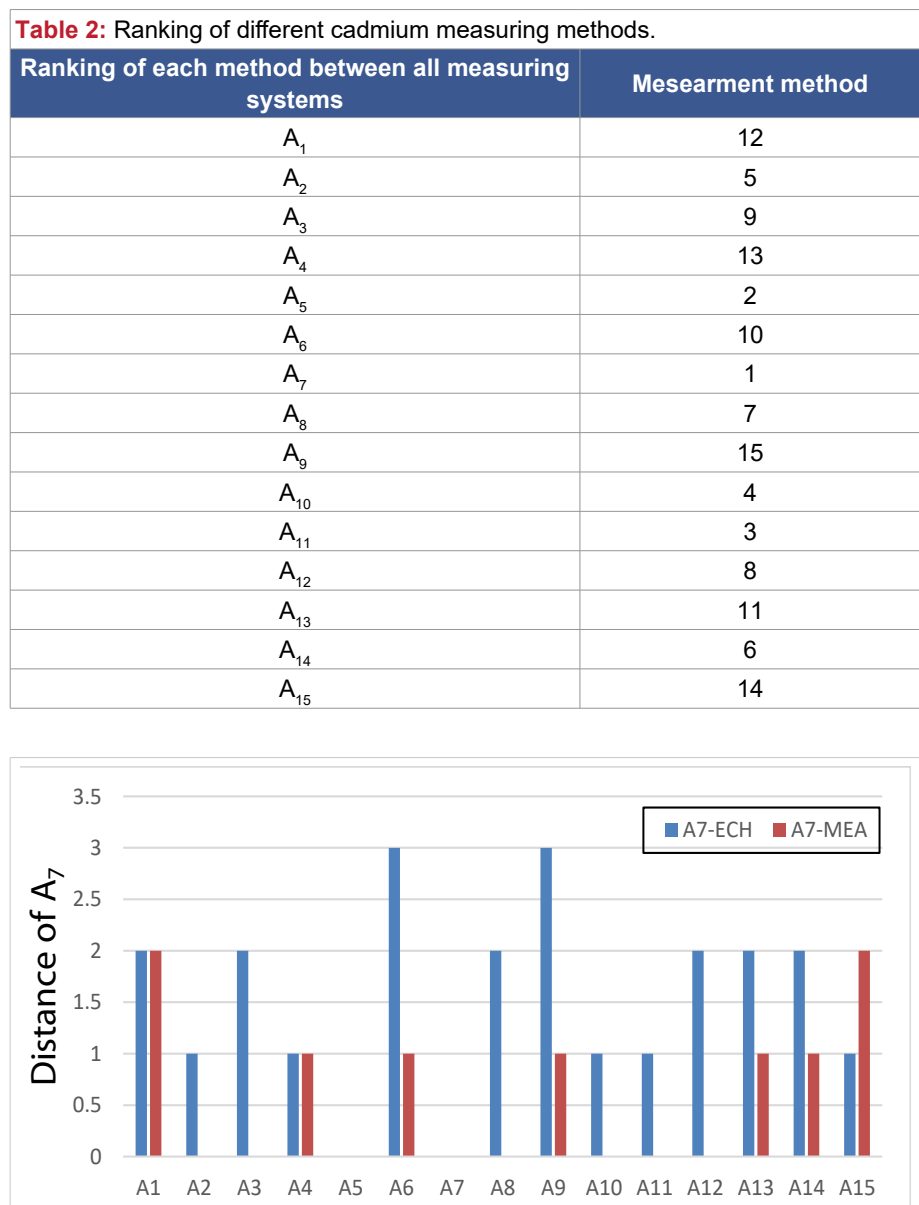

Selective Options for Cadmium Detection

Figure 2: The difference between the economic and accuracy indicators of the A7 option to the other options.

in the economic indicators (A7-ECH) and measurement accuracy (A7-MEA) is shown in Figure 2.

Considering Figure 2; The A7 and A5 optionsare significantly different from the other options in terms of economic and measurement accuracy. The difference between the A7 and A5 options is the measurement speed, which creates better conditions for the A7 rather than the A5. Therefore, option A7 was ranked first and option A5 placed next. Malek, et al. (A7) used the solid phase extraction method to measure lead and cadmium ions. In this method, after the reaction between cadmium and lead ions and dibenzyl dithiocarbamate ligand, Dowex Optipore V-493 resin was used to extract the desired complex. After extracting the complex on the resin surface, a solution of nitric acid in acetone was used to wash the resin. Finally, the eluent was injected into an atomic flame absorption device to measure cadmium and lead ions. Zhang, et al. (A5) also used a cloud point extraction method with a flame atomic absorption measurement system to measure small amounts of cadmium. In this method, cadmium with iodide anion produces $\mathrm{CdI} 4^{2-}$, which reacts with methyl green reagent to form a pair of non-polar ions that can be extracted in Triton X-114 surfactant. After centrifugation and separation of the organic phase from the aqueous one, the amount of cadmium in the sample is measured by atomic absorption spectroscopy. 


\section{Conclusion}

Cadmium is one of the toxic and allergenic metals that is found in the effluent of many industries, including machinery, plating, etc. Small amounts of this metal also have the possibility of biological accumulation and magnification in the body of living organisms and make them face various biological problems. Thus, the measurement of small amounts of cadmium, which is out of the accuracy range of measuring devices, becomes obvious. This study first conducted a library study of different methods for measuring small amounts of cadmium and prioritized these measurement methods using the ELECTRE analytical technique. The results of comparisons and analyzes showed that using Dowex Optipore V-493 resin and the extraction system in Triton $\mathrm{X}-114$ surfactant is more reliable than all other methods of measuring small amounts of cadmium.

\section{References}

1. Bahlsberg-Pahlsson AM. Toxicity of heavy metals $(\mathrm{Zn}, \mathrm{Cu}, \mathrm{Cd}, \mathrm{Pb})$ to vascular plants. Water Air Soil Pollut. 1989; 47: 287-319.

2. Kabata-Pendias A, Pendias H. Trace Element in Soil and Plants (3rd edition). CRC Press, Boca Raton, USA. 2001.

3. John R, Ahmad P, Gadgil K, Sharma S. Effect of cadmium and lead on growth, biochemical parameters and uptake in Lemna polyrrhiza $\mathrm{L}$. Plant Soil Environ. 2008; 54: 262-270.

4. Zazouli MA, Mohsein Bandpei A, Maleki A, Saberian M, Izanloo H Determination of cadmium and lead contents in black tea and tea liquor from Iran. Asia J Chem. 2010; 22: 1387-1393.

5. Li CC, Dang F, Cang L, Zhou DM, Willie JGM. Internal distribution of $\mathrm{Cd}$ in lettuce and resulting effects on $\mathrm{Cd}$ trophic transfer to the snail: Achatina fulica. Chemosphere. 2015; 135: 123-128.

PubMed: https://pubmed.ncbi.nlm.nih.gov/25930053/

6. Harak T, Kellner V, Ulk J, Jurkova M, Ejka P. Determination of Some Beer Flavors by Stir Bar Sorptive Extraction and Solvent Back Extraction. J Instit Brewing. 2007; 113: 154-158.

7. Pawliszyn J. Sample Preparation Quo Vadis, Anal Chem. 2003; 75:2543. PubMed: https://pubmed.ncbi.nlm.nih.gov/12948120/

8. Jahromi EZ, Bidari A, Assadi Y, Milani Hosseini MR, Jamali MR. Dispersive liquid-liquid microextraction combined with graphite furnace atomic absorption spectrometry: Ultra trace determination of cadmium in water samples. Analytica Chimica Acta. 2007; 585: 305-311.

9. Dadfarnia S, Haji Shabani AM, Kamranzadeh E, Talanta. 2009; 79 1061-1065.

10. Souza Dias FD, Bonsucesso JS, Alves LS, da Silva Filho DC, Costa ACS, et al. Development and optimization of analytical method for the determination of cadmium from mineral water samples by off-line solid phase extraction system using sisal fiber loaded TAR by FAAS. Microchemical J. 2013; 106: 363-367.

11. Abbasi S, Bahiraei A, Abbasi F. A highly sensitive method for simultaneous determination of ultra trace levels of copper and cadmium in food and water samples with luminol as a chelating agent by adsorptive stripping voltammetry. Food Chem. 2011; 129: 1274-1280. PubMed: https://pubmed.ncbi.nlm.nih.gov/25212367/

12. Xiang G, Wen S, Wu X, Jiang X, He L, et al. Food Chem. 2012; 132 : 532-536.

13. Li S, Cai S, Hu W, Chen H, Liu H. Ionic liquid-based ultrasound-assisted dispersive liquid-liquid microextraction combined with electrothermal atomic absorption spectrometry for a sensitive determination of cadmium in water samples. Spectrochimica Acta part B. 2009; 64: 666-671.

14. Melek E, Tuzen M, Soylak M. Flame atomic absorption spectrometric determination of cadmium(II) and lead(II) after their solid phase extraction as dibenzyldithiocarbamate chelates on Dowex Optipore V-493. Analytica Chimica Acta. 2006; 578: 213-219.

PubMed: https://pubmed.ncbi.nlm.nih.gov/17723714/

15. Parham H, Pourreza N, Rahbar N. Solid phase extraction of lead and cadmium using solid sulfur as a new metal extractor prior to determination by flame atomic absorption spectrometry. J Hazardous Materials. 2009; 163: 588-592.

16. Fang GZ, Tan J, Yan XP. An Ion-Imprinted Functionalized Silica Gel Sorbent Prepared by a Surface Imprinting Technique Combined with a Sol-Gel Process for Selective Solid-Phase Extraction of Cadmium(II). Anal Chem. 2005; 77: 1734-1739.

PubMed: https://pubmed.ncbi.nlm.nih.gov/15762579/

17. Tuzen M, Parlar K, Soylak M. Enrichment/separation of cadmium(II) and lead(II) in environmental samples by solid phase extraction. J Hazardous Materials. 2005; B121: 79-87.

PubMed: https://pubmed.ncbi.nlm.nih.gov/15885409/

18. Maranhao TA, Borges DLG, da Veiga MAMS, Curtius AJ. Cloud point extraction for the determination of cadmium and lead in biological samples by graphite furnace atomic absorption spectrometry. Spectrochimica Acta P Part B. 2005; 60: 667-672.

19. Mortada WI, Hassanien MM, Donia AF, Shokeir AA. Application of Cloud Point Extraction for Cadmium in Biological Samples of Occupationally Exposed Workers: Relation Between Cadmium Exposure and Renal Lesion. Biological Trace Element Res. 2015; 168: 303-310. PubMed: https://pubmed.ncbi.nlm.nih.gov/25998796/

20. Jafarvand S, Shemirani F. Anal Methods. 2011; 3: 1552-1560.

21. Zhang $\mathrm{C}$, Wang $\mathrm{Y}$, Chen $\mathrm{X}$, Xia H, Liang P. Determination of Cadmium and Lead in Human Teeth Samples Using Dispersive Liquid-liquid Microextraction and Graphite Furnace Atomic Absorption Spectrometry. J Chin Chem Soc. 2011; 58: 919-924.

22. Ishizaka A, Nemery P. Multi-Criteria Decision Analysis Methods and Software. John Willey \& Sons, Ltd. United Kingdom. 2013.

23. Sarı, U. Irem, Öztayşi B, Kahraman C. Fuzzy Analytic Hierarch Process Using Type-2 Fuzzy Set: An Application to Warehouse Location Selection. Multicriteria Decision Aid and Artificial Intelligence Links, Theory and Applications. John Willey\&Sons, Ltd. United Kingdom 9. 2013.

24. Triantaphyllou E. Multi-Criteia Decision Making Methods: A Comparative Study. Kluwer Academic Publishers, Boston, USA. 2000.

25. Triantaphyllou E. Multi-Criteria DecisionMaking Methods:A Comparative Study. (c) Springer Science+Business Media Dordrecht 2000.

26. BIAN KA. Application of Fuzzy AHP and ELECTRE to China Dry Port Location Selection. Asian J Shipping Logistics. 2011; 27: 331-353. 